Mitteilungen der Deutschen Gesellschaft

fïr Phlebologie

\title{
Berufsbegleitende Fortbildung Phlebologie - bitte bei den Ärztekammern anfragen
}

Die Prüfung zur Zusatzbezeichnung Phlebologie nach berufsbegleitender Weiterbildung scheint in den Ärztekammern angekommen zu sein. Interessenten sollten nun Kontakt zur zuständigen Ärztekammer aufnehmen und die Zulassung zur Prüfung / Kolloquium beantragen. Wichtig: Es müssen Gesprächsprotokolle von den Jahresgesprächen zwischen Weiterbilder und Prüf- ling vorgelegt werden, achten Sie also während der Weiterbildung darauf, auch diese zu führen und zu protokollieren.

Wir würden uns sehr freuen, wenn von der Möglichkeit, diese Prüfung abzulegen, reger Gebrauch gemacht wird, um unser Fach zu stärken und den Sinn dieser Zusatz- bezeichnung auch in den Kammern fortzuschreiben.

\section{IMPRESSUM}

Verantwortlich für Mitteilungen der DGP:

Dr. med. Erika Mendoza 